\title{
The operation threshold of a double barrier phonon laser
}

\author{
I. Camps ${ }^{\text {a,b,1 }}$ S. S. Makler ${ }^{\text {c,b }}$ \\ a Physics Faculty - IMRE, University of Havana, Cuba \\ ${ }^{\mathrm{b}}$ Instituto de Física, Universidade Federal Fluminense, Niterói, RJ, Brazil \\ ${ }^{\mathrm{c}}$ Instituto de Física, Universidade Estadual do Rio de Janeiro, RJ, Brazil
}

\begin{abstract}
We make an adaptation of laser modelling equations to describe the behavior of a phonon laser (saser). Our saser consists of an AlGaAs/GaAs double barrier heterostructure designed to generate an intense beam of transversal acoustic $(T A)$ phonons. To study our system, we begin with a Hamiltonian that describes the decay of primary longitudinal optical phonons $\left(L O_{1}\right)$ into secondary $\left(L O_{2}\right)$ and $T A$ $\left(\mathrm{LO}_{1} \rightarrow \mathrm{LO}_{2}+\mathrm{TA}\right)$ and its inverse process (recombination). Using this Hamiltonian, a set of coupled equations of motion for the phonons is obtained. We also consider the interaction between the phonons and its reservoirs. These interactions are introduced in the equations of motion leading to a set of coupled Langevin equations. In order to obtain an expression to describe our saser we apply, in the Langevin equations, an adiabatic elimination of some variables of the subsystem. Following the method above we obtain the value of the injection threshold for the operation of our phonon laser. At this threshold occurs a phase transition from a disordered to a coherent state. It is shown that it is not necessary a big "optical" pumping to get a sasing region.
\end{abstract}

Key words: A. nanostructures, A. quantum wells, A. semiconductors, D. anharmonicity, D. phonons.

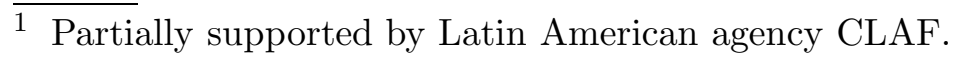

Preprint submitted to Elsevier Preprint

5 November 2018 


\section{Introduction}

Sasers are devices capable of producing beams of coherent phonons. Besides the system considered here, several other kinds of such devices were proposed and constructed [1-7].

In recent works [8-13] the kinetics and the dynamical properties of the double barrier phonon laser were discussed. In this paper we study the system from a point of view close to that used in quantum optics to study the onset of the lasing regime. In spite of the fact that the system is far from equilibrium and thus concepts like temperature and chemical potential can not be applied, the process is analogous to a phase transition $[14,15]$. This transition is not governed by temperature but an energy flux (in this case by the injection rate of primary longitudinal optical $L O_{1}$ phonons). We were able to obtain the potential that describes the states of our system and consequently, the threshold value for the coherent transversal acoustic $(T A)$ phonon emission.

The paper is organized as follows. A description of the saser device is presented in section 2. The multimode case is analyzed in section 3 . In section 4 the potential that describes our system is obtained. Finally, in section 5 we present our results and conclusions.

\section{The system}

The phonon laser studied here consists of an AlGaAs/GaAs double-barrier heterostructure $(\mathrm{DBH})$. This device was designed in such a way that, for a small applied bias $V$, the difference $\Delta \varepsilon$ between the first excited level $\varepsilon_{1}$ and the ground state $\varepsilon_{0}$ in the well is less than the longitudinal optical $\left(L O_{1}\right)$ phonon energy $\hbar \omega_{1}$. For a greater bias the resonant condition $\Delta \varepsilon \approx \hbar \omega_{1}$ is achieved and the electrons begin to decay to the ground state by emitting primary $L O_{1}$ phonons. For an $A l$ concentration greater than 0.25 [16] or 0.3 [17], these phonons are confined inside the well (they can be also absorbed by exciting electrons from $\varepsilon_{0}$ to $\left.\varepsilon_{1}\right)$.

The process described above, acts in parallel with the decay of primary $L O_{1}$ phonons due to anharmonicity. One of the products of this decay is a secondary longitudinal optical phonon $\left(\mathrm{LO}_{2}\right)$, the other is a $\mathrm{TA}$ phonon [18]. The $\mathrm{LO}_{2^{-}}$ $T A$ pair is produced by stimulated emission. Therefore these $T A$ phonons could be coherent and form a beam that it is called saser by analogy with a laser.

The DBH saser beam could be applied to acoustic nanoscopy, phonoelectronics 
and phonolitography. This was discussed in more detail in a previous article [8].

The potential profile and the level positions at the resonant condition are shown in figure 1.

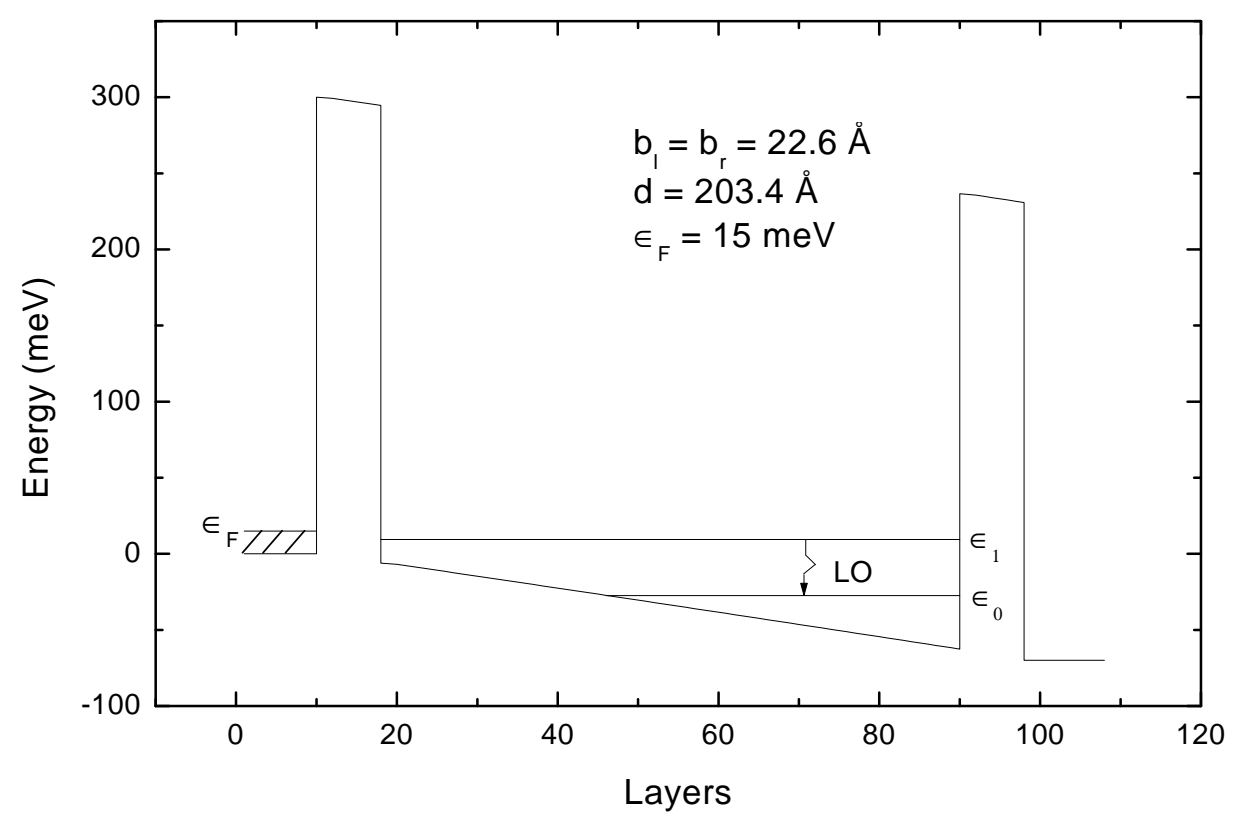

Fig. 1. Potential profile and energy levels at resonant condition. $b_{l}, b_{r}, d$ and $\epsilon_{F}$ are respectively the barriers and well widths and the Fermi level at the emitter.

\section{The multimode case}

The fundamental mechanism in this saser is the generation of the $T A$ phonons due to the decay of primary $L O_{1}$ phonons. Therefore, the other processes (i.e., emission-absorption of the $L O_{1}$ phonon through electron transitions and the $\mathrm{LO}_{2}$ phonon decay) are secondary and are taken into account just as a result of the interaction of each phonon with its reservoir.

As the $T A$ phonon emission results from the decay of $L O_{1}$ phonons, they could be emitted in many modes, corresponding to the different values of its wave vector $\mathbf{q}_{3}$ parallel to the interface.

The number $N_{\mathbf{q}_{3}}$ of wave vectors parallel to the interface can be estimated as $N_{\mathbf{q}_{3}} \sim S_{0} / a^{2}$, where $S_{0}$ is the device area and $a$ is the lattice parameter. For the values used here, $S_{0}=0.5 \cdot 10^{-3} \mathrm{~mm}^{2}$ and $a=2.825 \AA, N_{\mathbf{q}_{3}}$ is of the order 
of $7 \cdot 10^{9}$. The total number of emitted $T A$ phonons is $\sim 2 \cdot 10^{4}$ [13], which means that, at the beginning of the phonon emission, the number of phonons per wave vector $\mathbf{q}_{3}$ is $\sim 3 \cdot 10^{-6}$. As the applied bias increases, the emission in a particular mode called $\mathbf{q}_{3}^{0}$ grow slowly until a certain threshold value $V=V_{t h}$ is reached. For values of $V$ greater than $V_{t h}$, the phonons in that mode are emitted in a coherent way implying that the population $n_{\mathbf{q}_{3}^{0}}$ is the only one macroscopically non zero. In this section, the possibility of macroscopically coexistence of more than one mode is studied.

\subsection{The Hamiltonian}

The Hamiltonian that describes our system can be written as:

$$
\begin{gathered}
\mathcal{H}=\hbar \omega_{1} \sum_{\mathbf{q}_{1}} b_{\mathbf{q}_{1}}^{\dagger} b_{\mathbf{q}_{1}}+\hbar \omega_{2} \sum_{\mathbf{q}_{2}} b_{\mathbf{q}_{2}}^{\dagger} b_{\mathbf{q}_{2}}+\hbar \omega_{3} \sum_{\mathbf{q}_{3}} b_{\mathbf{q}_{3}}^{\dagger} b_{\mathbf{q}_{3}}+ \\
\hbar \gamma \sum_{\mathbf{q}_{1}, \mathbf{q}_{2}, \mathbf{q}_{3}}\left(b_{\mathbf{q}_{1}}^{\dagger} b_{\mathbf{q}_{2}} b_{\mathbf{q}_{3}}+b_{\mathbf{q}_{1}} b_{\mathbf{q}_{2}}^{\dagger} b_{\mathbf{q}_{3}}^{\dagger}\right)
\end{gathered}
$$

where $\mathbf{q}_{1}, \mathbf{q}_{2}$ and $\mathbf{q}_{3}$ are respectively the $L O_{1}, L O_{2}$ and $T A$ wave vectors parallel to the interfaces, and must satisfy $\mathbf{q}_{1}=\mathbf{q}_{2}+\mathbf{q}_{3}, \hbar \omega_{i}(i=1,2,3)$ are the energies of each kind of phonons and $\gamma$ is the phonon-phonon interaction coefficient.

\subsubsection{The Langevin equations}

The equations of motion for the operators $n_{\mathbf{q}_{1}}\left(n_{\mathbf{q}_{i}}=b_{\mathbf{q}_{i}}^{\dagger} b_{\mathbf{q}_{i}}\right), b_{\mathbf{q}_{2}}, b_{\mathbf{q}_{3}}$ are obtained from the Hamiltonian (1). We remark that the saser system is coupled to reservoirs. The $L O_{1}$ phonons are coupled through the emission and absorption processes to the electron system. In the case of the $\mathrm{LO}_{2}$ phonons, they are coupled to the other phonon modes to which they decay, whereas the $T A$ phonons are connected to the other $T A$ phonon modes outside the well. Thus the total Hamiltonian includes, besides the terms shown in equation (1), the interaction with the reservoirs. By using the methods described in references $[19,20]$, the reservoir coordinates can be eliminated leading to the following Langevin equations

$$
\begin{aligned}
& \frac{d n_{\mathbf{q}_{1}}}{d t}=-i \gamma \sum_{\mathbf{q}_{2}, \mathbf{q}_{3}}\left\{b_{\mathbf{q}_{1}}^{\dagger} b_{\mathbf{q}_{2}} b_{\mathbf{q}_{3}}-b_{\mathbf{q}_{1}} b_{\mathbf{q}_{2}}^{\dagger} b_{\mathbf{q}_{3}}^{\dagger}\right\}+G_{\mathbf{q}_{1}}-\Gamma_{1} n_{\mathbf{q}_{1}}+\hat{F}_{1} \\
& \frac{d b_{\mathbf{q}_{2}}}{d t}=-\left(i \omega_{2}+\kappa_{2}\right) b_{\mathbf{q}_{2}}-i \gamma \sum_{\mathbf{q}_{1}, \mathbf{q}_{3}} b_{\mathbf{q}_{1}} b_{\mathbf{q}_{3}}^{\dagger}+\hat{F}_{2},
\end{aligned}
$$




$$
\frac{d b_{\mathbf{q}_{3}}}{d t}=-\left(i \omega_{3}+\kappa_{\mathbf{q}_{3}}\right) b_{\mathbf{q}_{3}}-i \gamma \sum_{\mathbf{q}_{1}, \mathbf{q}_{2}} b_{\mathbf{q}_{1}} b_{\mathbf{q}_{2}}^{\dagger}+\hat{F}_{3},
$$

where $G_{\mathbf{q}_{1}}$ and $\Gamma_{1}$ are the emission and absorption rates for the $L O_{1}$ phonons due the interaction with its reservoir (i.e., the electrons), $\kappa_{2}$ is the decay rate of $\mathrm{LO}_{2}$ phonons, $\kappa_{\mathbf{q}_{3}}$ is the escape rate of the $T A$ phonons and $\hat{F}_{i}(i=$ $1,2,3)$ are the fluctuation forces. The $T A$ phonons escape rate is different for each mode, thus depending on $\mathbf{q}_{3}$. As we are not interested in studying the statistical properties of the saser, in the following the fluctuation forces will not be considered.

In order to obtain only one equation for the $T A$ phonons, we make the adiabatic approximation in equations (2) and (3). First we replace $b_{\mathbf{q}_{1}}(t)=$ $\tilde{b}_{\mathbf{q}_{1}}(t) e^{-i \omega_{1} t}$ and $b_{\mathbf{q}_{3}}(t)=\tilde{b}_{\mathbf{q}_{3}}(t) e^{-i \omega_{3} t}$ in $(3)$. Secondly, we integrate $(3)$ explicitly to get

$$
b_{\mathbf{q}_{2}}=-i \gamma \sum_{\mathbf{q}_{1}, \mathbf{q}_{3}} \int_{-\infty}^{t} e^{-\left[i\left(\omega_{1}-\omega_{2}-\omega_{3}\right)+\kappa_{2}\right](t-\tau)}\left(\tilde{b}_{\mathbf{q}_{1}} \tilde{b}_{\mathbf{q}_{3}}^{\dagger}\right)_{\tau} d \tau
$$

Assuming that the relaxation time of the $b_{\mathbf{q}_{2}}$ phonons is much smaller than the relaxation of $\tilde{b}_{\mathbf{q}_{1}}$ and $\tilde{b}_{\mathbf{q}_{3}}^{\dagger}$, we can take $\tilde{b}_{\mathbf{q}_{1}} \tilde{b}_{\mathbf{q}_{3}}^{\dagger}$ out of the integral in equation (5) obtaining

$$
b_{\mathbf{q}_{2}}=-i \gamma \sum_{\mathbf{q}_{1}, \mathbf{q}_{3}} \frac{e^{-i\left(\omega_{1}-\omega_{3}\right) t}}{-i \nu+\kappa_{2}} \tilde{b}_{\mathbf{q}_{1}} \tilde{b}_{\mathbf{q}_{3}}^{\dagger}
$$

where $\nu=\omega_{1}-\omega_{2}-\omega_{3}$.

In a similar way, we obtain for $n_{\mathbf{q}_{1}}$

$$
n_{\mathbf{q}_{1}}=\frac{G_{\mathbf{q}_{1}}}{\Gamma_{1}}\left(1-\frac{\Omega}{\Gamma_{1}} \sum_{\mathbf{q}_{3}} n_{\mathbf{q}_{3}}\right)
$$

where

$$
\Omega=\frac{2 \gamma^{2} \kappa_{2}}{\nu^{2}+\kappa_{2}^{2}}
$$

Finally, we replace (6) and (7) in (4) and we get, after some manipulations, an equation for the $T A$ phonon populations

$$
\frac{d n_{\mathbf{q}_{3}}}{d t}=\left(\Omega n_{1}-2 \kappa_{\mathbf{q}_{3}}\right) n_{\mathbf{q}_{3}},
$$


where $n_{1}=\sum_{\mathbf{q}_{1}} n_{\mathbf{q}_{1}}$.

Considering as an example only two modes, $\mathbf{q}_{3}^{0}$ and $\mathbf{q}_{3}^{1}$, we get for the stationary case of equation (8):

$$
\begin{gathered}
n_{3}^{0}\left(\Omega n_{1}-2 \kappa_{3}^{0}\right)=0 \\
n_{3}^{1}\left(\Omega n_{1}-2 \kappa_{3}^{1}\right)=0
\end{gathered}
$$

If both modes would be present, $\left(n_{3}^{0} \neq 0\right.$ and $\left.n_{3}^{1} \neq 0\right)$ both parenthesis in (9) and (10) would be zero. This is a contradiction (because $\kappa_{3}^{0} \neq \kappa_{3}^{1}$ ). This contradiction only can be solved if just one mode is present and the other one has died out. Therefore, only a single mode, the one with largest lifetime inside the well and closest to resonance, survives. The former analysis also can be done quite rigorously for many modes.

When the $L O_{1}$ decay, the $T A$ phonons are emitted in the [111] direction [21]. The device is grown in this direction, such that the $T A$ phonon beam will make multiple reflections between the walls of the well, implying in large lifetime for these phonons. Indeed, for a small applied bias, the $T A$ phonons are emitted with any wave vector $\mathbf{q}_{3}$. In this regime, the device works as a sound emitting diode (SED). With a further increase in the applied potential, when a certain value of $V$ is reached, the $T A$ phonon distribution on $\mathbf{q}_{3}$ become more and more sharp giving place to a phase transition. In this case $n_{3}^{0}$ grows suddenly from about $10^{-6}$ to about $10^{4}$. Thus, a great number of $T A$ phonons would have only one mode: $\mathbf{q}_{3}^{0}=0$ (which imply $\mathbf{q}_{1}=\mathbf{q}_{2}$ ). This mode will survive and slave the others, making the system to self-organize and emit just in this mode.

\section{The single mode case}

In order to get the threshold for which $n_{3}^{0}$ begins to be macroscopically non zero, we must first obtain an equation for the amplitude of the single mode $b_{3}^{0}$ that slaves the system. To do that, we make the same approximations as described in the previous section. The equation obtained for $b_{3}^{0}$ is

$$
\frac{d b_{3}}{d t}=\left(\frac{\gamma^{2}}{\Gamma_{1} \kappa_{2}} G_{1}-\kappa_{3}\right) b_{3}^{0}-2 G_{1}\left(\frac{\gamma^{2}}{\Gamma_{1} \kappa_{2}}\right)^{2} b_{3}^{0 \dagger} b_{3}^{0} b_{3}^{0}
$$

where $G_{1}=\sum_{\mathbf{q}_{1}} G_{\mathbf{q}_{1}}$. 
The right-hand side of (11) can be obtained from the potential:

$$
U_{c}\left(b_{3}^{0}\right)=\alpha\left|b_{3}^{0}\right|^{2}+\beta\left|b_{3}^{0}\right|^{4},
$$

with

$$
\alpha=\kappa_{3}-\frac{\gamma^{2}}{\Gamma_{1} \kappa_{2}} G_{1}, \quad \beta=G_{1}\left(\frac{\gamma^{2}}{\Gamma_{1} \kappa_{2}}\right)^{2}
$$

such that

$$
\frac{d b_{3}^{0}}{d t}=-\frac{d U_{c}\left(b_{3}^{0}\right)}{d b_{3}^{\dagger}}
$$

The expressions for the parameters $G_{1}$ and $\Gamma_{1}$ are [13]

$$
\Gamma_{1}=w\left(N_{0}-N_{1}\right), \quad G_{1}=w N_{1} .
$$

where $w$ is the electron transition rate and $N_{0}$ and $N_{1}$ are respectively the electron populations of the fundamental and excited levels.

The shape of the potential (12) is similar to that obtained in the GinzburgLandau phase transition theory. For $\alpha>0$ the potential has a minimum at $n_{3}^{0}=0\left(n_{3}^{0}=\left|b_{3}^{0}\right|^{2}\right)$. In the case of $\alpha<0$, the minimum is obtained for $n_{3}^{0} \neq 0$. The transition from $\alpha>0$ to $\alpha<0$ is due to the competition between

processes of gain, with a rate $G_{1} \frac{\gamma^{2}}{\Gamma_{1} \kappa_{2}}$ and losses, with a rate $\kappa_{3}$. The threshold is attained when $\alpha=0$, i.e., $G_{1}=\frac{\Gamma_{1} \kappa_{2} \kappa_{3}}{\gamma^{2}}$.

\section{Results and Conclusions}

The parameters used in our calculation are: the escape rate of $T A$ phonons $\kappa_{3}^{0}=0.05 \mathrm{ps}^{-1}$, estimated from its group velocity near the $L$ point of the Brillouin zone, the decay rate of the $\mathrm{LO}_{2}$ phonons $\kappa_{2}=20 \mathrm{ps}^{-1}$ (by assuming a small stimulated decay) and the phonon-phonon interaction coefficient $\gamma=0.5 \mathrm{ps}^{-1}$ calculated in reference [22] following the work of Klemens [23]. The decay rate $\gamma_{0}$ obtained from this value is in agreement with the experimental result of Vallée [18]. The values of $G_{1}$ and $\Gamma_{1}$ depend on the $L O_{1}$ phonon emission rate $w$ and on the electron populations $N_{0}$ and $N_{1}$ that were calculated in reference [13] by solving the kinetic equations.

The total generation rate of the $L O_{1}$ phonons $G_{1}$, depends on the applied bias $V$ (i.e., it turns out from electron transitions that are $V$ dependent [13]). 
Thus, the applied bias is the external parameter that control the intensity of the saser emission.

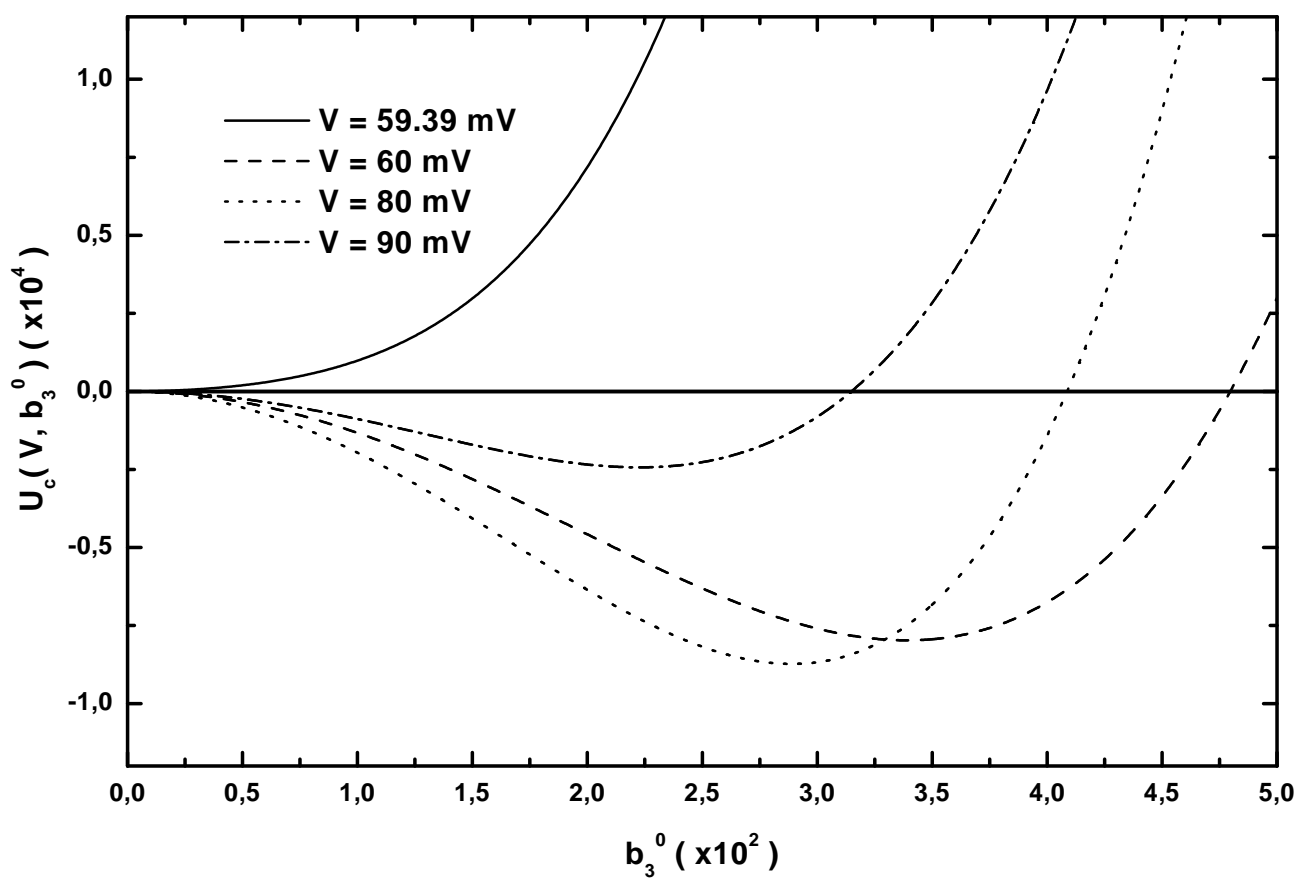

Fig. 2. The potential $U_{c}$ as a function of the $T A$ phonon amplitude for different values of the applied bias.

In figure 2, the potential (12) is plotted as a function of the $T A$ phonon amplitude for different values of the applied bias $V$. As it can be seen, for $V>V_{t h}=59.4 \mathrm{mV}$ this potential has different minima for different values of $V$. These minima represent the stable points $b_{3}^{s}$ of the system.

In figure 3 the stable points are plotted as function of $V$. The solid line represents the $n_{3}^{s}$ calculated in this work. The dashed line is the $T A$ phonon population calculated in a previous work from the kinetic equations [13].

Figure 4 shows a comparison in the region where the $T A$ generation begins (dashed region in figure 3). From this comparison two conclusions arise. First, as it can be seen (solid line), the $T A$ coherent emission does not start until a certain threshold of the applied bias is reached. At $V_{t h}$ the $L O_{1}$ emission rate $G_{1}$ is of the order of $279 \mathrm{ps}^{-1}$ and the total $T A$ phonon population $n_{3}$ is $\sim 4658$, whereas $n_{3}^{s}=0$. The $T A$ phonons generated for a bias greater than $V_{t h}$ are coherent. Secondly, our previous calculations [13], even without considering the self-organization process, gave results that agree quite well with those presented here. This results show that the coherent emission occurs immediately after the condition of $\mathrm{LO}_{1}$ phonon emission is reached. That means that it is not necessary a big "optical" pumping to get a sasing region. In 


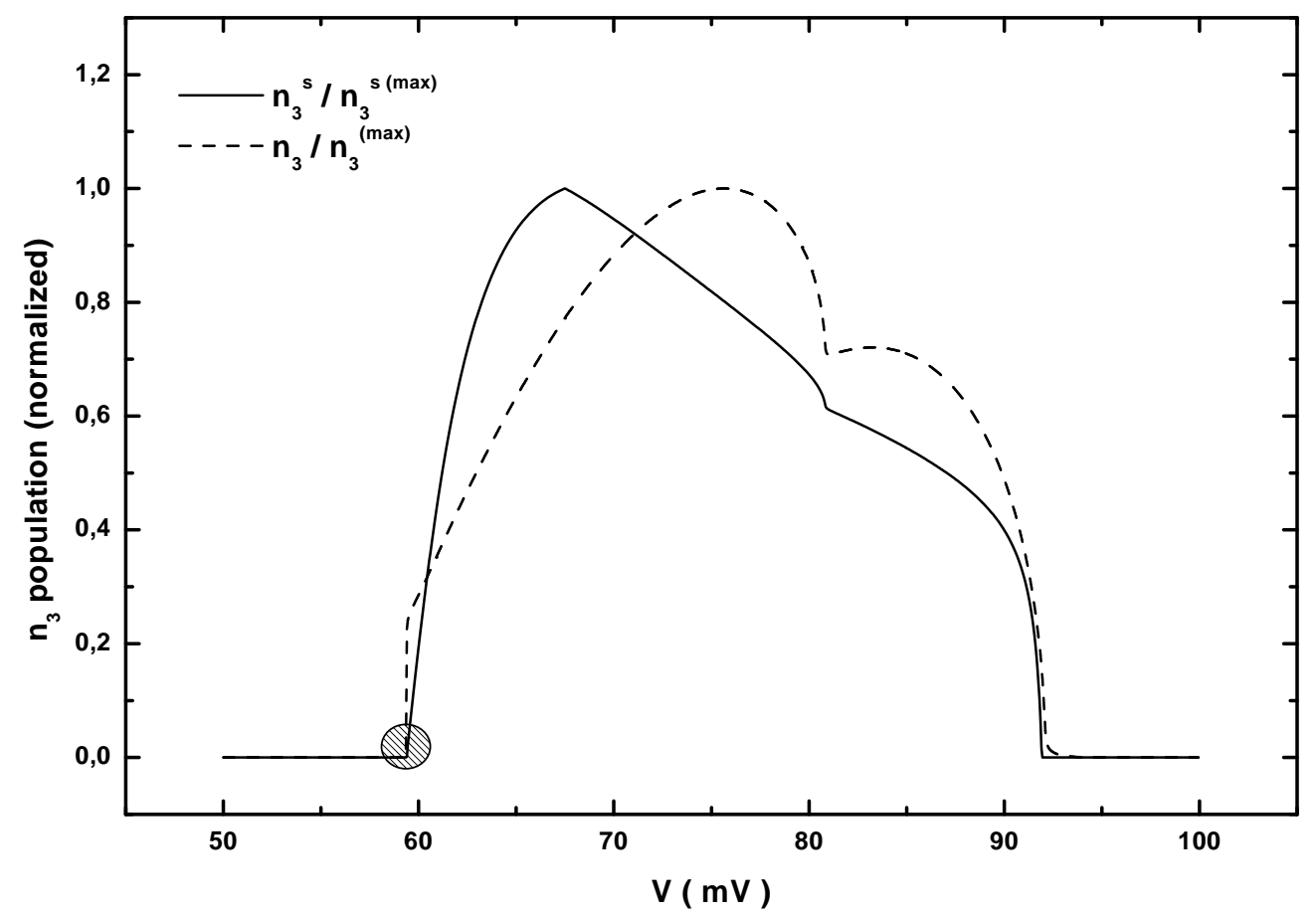

Fig. 3. The solid line shows the result for the coherent mode population obtained here (the minima of the previous figure), whereas the dashed line shows the total $T A$ phonon population obtained from the kinetic equations [13].

summary, we show in this work that the $T A$ phonons, produced by stimulated emission, constitute a coherent phonon beam in the parameter region discussed above. 


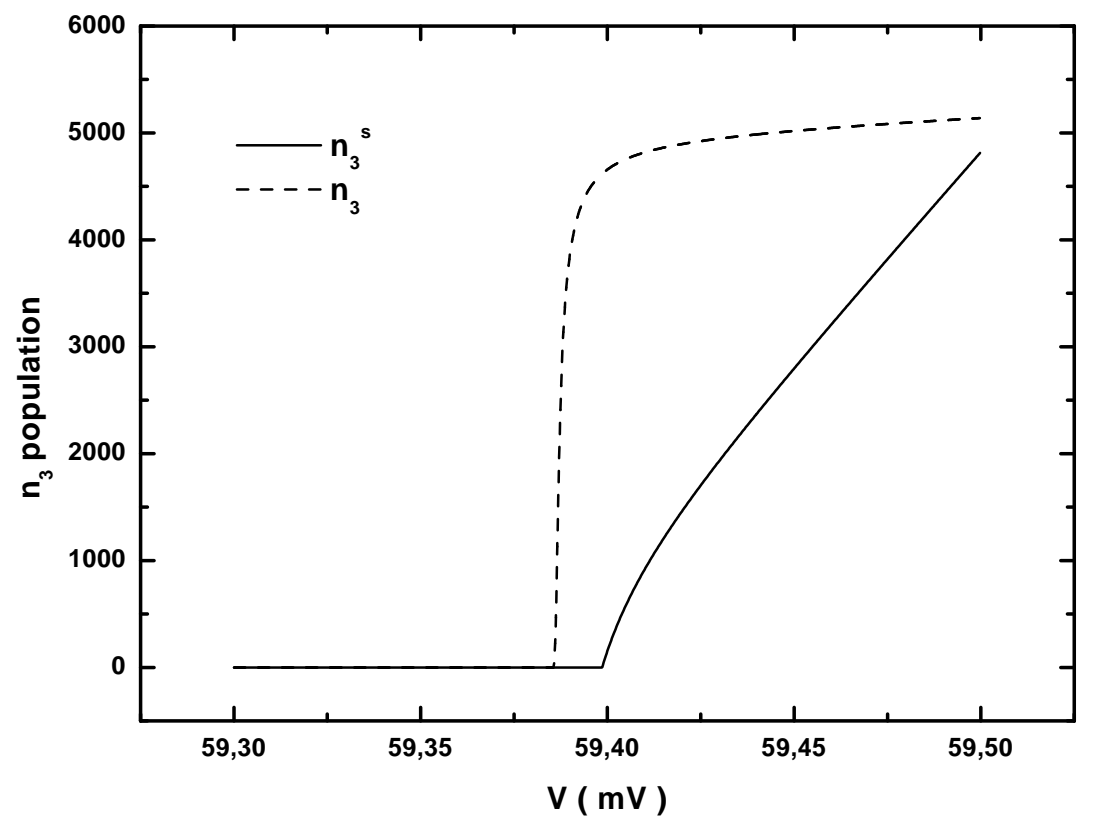

Fig. 4. The dashed region in figure 3 is shown in detail. We can see that the $T A$ emission process begins incoherent. It becomes coherent when the injection rate of phonons $L O_{1}$ is $G_{1} \geq \frac{\Gamma_{1} \kappa_{2} \kappa_{3}}{\gamma^{2}}$.

\section{References}

[1] P. A. Fokker, J. I. Dijkhuis, and H. W. de Wijn, "Stimulated emission of phonons in an acoustical cavity," Phys. Rev. B, vol. 55, no. 5, pp. 2925 - 2933, Feb. 1997.

[2] P. A. Fokker, R. S. Meltzer, Y. P. Wang, J. I. Dijkhuis, and H. W. de Wijn, "Suppression of stimulated phonon emission in ruby by a magnetic-field gradient," Phys. Rev. B, vol. 55, no. 5, pp. 2934 - 2937, Feb. 1997.

[3] J. Y. Prieur, R. Höhler, J. Joffrin, and M. Devaud, "Sound amplification by stimulated emission of radiation in an amorphous compound," Europhys Lett., vol. 24, no. 5, pp. 409 - 414, Nov. 1993.

[4] J. Y. Prieur, M. Devaud, J. Joffrin, C. Barre, and M. Stenger, "Sound amplification by stimulated emission of phonons using two-level systems in glasses," Physica B, vol. 220, pp. 235 - 238, Apr. 1996.

[5] S. T. Zavtrak, "Acoustic laser with dispersed particles as an analog of a freeelectron laser," Phys. Rev. E, vol. 51, no. 3, pp. 2480 - 2484, Mar. 1995.

[6] S. T. Zavtrak, "Acoustical laser with mechanical pumping," J. Acoust. Soc. Amer., vol. 99, no. 2, pp. 730 - 733, Feb. 1996. 
[7] Andrew Watson, "Pump up the volume," New Scientist, pp. 37 - 40, Mar. 1999.

[8] S. S. Makler, M. I. Vasilevskiy, E. V. Anda, D. E. Tuyarot, J. Weberszpil, and H. M. Pastawski, "A source of terahertz coherent phonons," J. of Phys. Cond. Matt., vol. 10, no. 26, pp. 5905 - 5921, Jul. 1998.

[9] S. S. Makler, D. E. Tuyarot, E. V. Anda, and M. I. Vasilevskiy, "Ultra-highfrequency coherent sound generation in resonant tunneling," Surf. Sci., vol. 362, no. 1-3, pp. 239 - 242, 1996.

[10] D. E. Tuyarot, S. S. Makler, E. V. Anda, and M. I. Vasilevskiy, "Double-barrier coherent sound generator: a new device," Superlatt. Microstruct., vol. 22, no. 3, pp. $427-430,1997$.

[11] J. Weberszpil, S. S. Makler, E. V. Anda, and M. I. Vasilevskiy, "A many body study of the SASER dynamics," Microelectron. Eng., vol. 43 - 44, pp. $471-$ 479, Aug. 1998.

[12] I. Camps, S. S. Makler, and E. V. Anda, "A quantum formalism for a terahertz acoustic laser," Braz. J. of Phys., vol. 29, no. 4, pp. 694 - 701, 1999.

[13] S. S. Makler, I. Camps, J. Weberszpil, and D. E. Tuyarot, "A double-barrier heterostructure generator of terahertz phonons: many-body effects," J. of Phys. Cond. Matt., vol. 12, no. 13, pp. 3149 - 3172, Apr. 2000.

[14] H. Haken, "Cooperative phenomena in systems far from thermal equilibrium and in nonphysical systems," Rev. Mod. Phys., vol. 47, no. 1, pp. $67-121$, Jan. 1975.

[15] H. Haken, Synergetics. An Introduction. Nonequilibrium Phase Transitions and Self-Organization in Physics, Chemistry and Biology, Springer Series in Synergetics. Springer-Verlag, Berlin, third edition, 1983.

[16] B. Jusserand, F. Mallot, J. M. Moison, and G. Leroux, "Atomic-scale roughness of GaAs/AlAs interfaces. A Raman-scattering study of asymmetrical shortperiod superlattices," Appl. Phys. Lett., vol. 57, no. 6, pp. 560 - 562, Aug. 1990.

[17] J. M. Jacob, D. M. Kim, A. Bouchalkha, J. J. Sony, J. F. Klem, H. Hou, C. W. Tu, and H. Morkoç, "Spatial characteristic of GaAs, GaAs-like, and AlAs-like LO phonons in GaAs- $A l_{x} G a_{1-x} A s$ superlattices. The strong x-dependence," Sol. State Comm., vol. 91, no. 9, pp. 721 - 724, Sep. 1994.

[18] F. Vallée and F. Bogani, "Coherent time-resolve investigation of LO-phonon dynamics in GaAs," Phys. Rev. B, vol. 43, no. 14, pp. 12049 -12 052, May 1991.

[19] Claude Cohen-Tannoudji, Jacques Dupont-Roc, and Gilbert Grynberg, AtomPhoton Interactions, John Wiley \& Sons, Inc., New York, 1992.

[20] Ulrich Weiss, Quantum dissipative systems, vol. 10 of Series in Modern Condensed Matter Physics, World Scientific Publishing, P O Box 128, Farrer Road, Singapore 912805, second edition, 1999. 
[21] F. Vallée, "Time resolved investigation of coherent LO-phonon relaxation in III-V semiconductors," Phys. Rev. B, vol. 49, no. 4, pp. 2460 - 2468, Jan. 1994.

[22] I. Camps and S. Makler, "The terahertz phonon laser: a full quantum treatment," submitted for publication.

[23] P. G. Klemens, "Anharmonic decay of optical phonons," Phys. Rev., vol. 148, no. 2, pp. 845 - 848, Aug. 1966. 